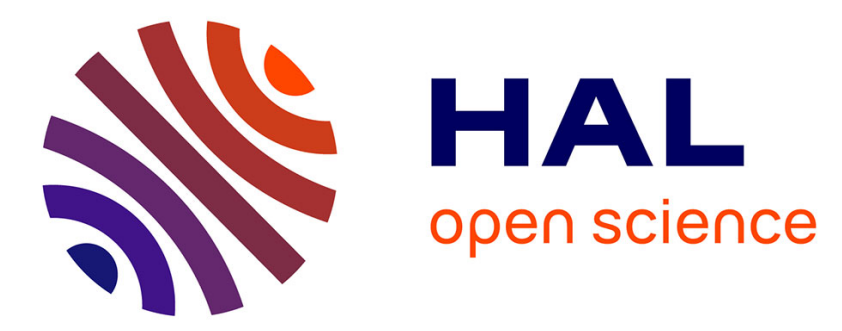

\title{
Determination of Interfacial Concentration of a Contaminated Droplet from Shape Oscillation Damping
}

Benjamin Lalanne, Olivier Masbernat, Frédéric Risso

\section{To cite this version:}

Benjamin Lalanne, Olivier Masbernat, Frédéric Risso. Determination of Interfacial Concentration of a Contaminated Droplet from Shape Oscillation Damping. Physical Review Letters, 2020, 124 (19), pp.0. 10.1103/PhysRevLett.124.194501 . hal-02641224

\section{HAL Id: hal-02641224 \\ https://hal.science/hal-02641224}

Submitted on 28 May 2020

HAL is a multi-disciplinary open access archive for the deposit and dissemination of scientific research documents, whether they are published or not. The documents may come from teaching and research institutions in France or abroad, or from public or private research centers.
L'archive ouverte pluridisciplinaire $\mathbf{H A L}$, est destinée au dépôt et à la diffusion de documents scientifiques de niveau recherche, publiés ou non, émanant des établissements d'enseignement et de recherche français ou étrangers, des laboratoires publics ou privés. 


\section{OATAO \\ Open Archive Toulouse Archive Ouverte}

\section{Open Archive Toulouse Archive Ouverte}

OATAO is an open access repository that collects the work of Toulouse researchers and makes it freely available over the web where possible

This is an author's version published in: http://oatao.univ-toulouse.fr/ 26004

Official URL : https://doi.org/10.1103/PhysRevLett.124.194501

\section{To cite this version:}

Lalanne, Benjamin $\leftrightarrows$ and Masbernat, Olivier Determination of Interfacial Concentration of a Contaminated Droplet from Shape Oscillation Damping. (2020) Physical Review Letters, 124 (19). ISSN 0031-9007

Any correspondence concerning this service should be sent to the repository administrator: tech-oatao@listes-diff.inp-toulouse.fr 


\title{
Determination of Interfacial Concentration of a Contaminated Droplet from Shape Oscillation Damping
}

\author{
Benjamin Lalanne $\odot^{*}$ and Olivier Masbernat $\odot$ \\ Laboratoire de Génie Chimique, Université de Toulouse, CNRS, INPT, UPS, Toulouse, France \\ and FR FERMAT, Université de Toulouse, CNRS, INPT, INSA, UPS, Toulouse, France \\ Frédéric Risso \\ Institut de Mécanique des Fluides de Toulouse (IMFT), Université de Toulouse, CNRS, Toulouse, France \\ and FR FERMAT, Université de Toulouse, CNRS, INPT, INSA, UPS, Toulouse, France
}

\begin{abstract}
We present a method to measure the very small interfacial concentration of a contaminant that is irreversibly adsorbed on the interface of a bubble or droplet. It is an application of the linear theory of shape oscillation which relates the Gibbs elasticity to the damping, extended by numerical simulations to deal with moving droplets. It explains previous unexpected observations on the effect of contamination at various oscillation wavelengths. The experimental procedure is easy to implement and can thereby deeply enhance the analysis of most systems involving uncontrolled contamination.
\end{abstract}

DOI: 10.1103/PhysRevLett.124.194501

Multiphase flows with drops or bubbles are widely encountered in industrial processes (chemical reactors, extraction columns, spraying and combustion devices) as well as in soft materials and biological applications (advanced materials, medicine), the governing physical phenomena at droplet scale being generally investigated through laboratory dedicated experiments. An important issue associated to these flows is the level of interface contamination due to chemical or biochemical impurities. Even if they are present in residual concentration, such contaminants have a drastic impact on the two-phase flow dynamics. Several hydrodynamics features can be strongly altered such as bubble-bubble interactions [1] or the rates of transfer across the interfaces [2]. In particular, the Marangoni stresses at a drop surface that result from buoyancy-induced motion strongly reduce the settling velocity as compared to clean conditions [3-7]. The presence of adsorbed contaminants leads generally to discrepancy between experimental observations and simulation predictions [8,9], making difficult the establishment of reliable predictive laws. However, measuring minute interfacial concentrations of a droplet is challenging and beyond the reach of previous experimental techniques. This Letter demonstrates that it is possible to measure the Gibbs elasticity of a rising droplet from the analysis of the free shape oscillations, which naturally take place when a nonspherical droplet is released from rest, and to jointly access the value of the interfacial tension. Then, the surface concentration can be estimated. The strength of this method lies in the fact that the oscillation damping rate is highly sensitive to small variations of surface concentration.
Let us review existing experimental methods of surface concentration assessment. Methods relying on the measurement of the interfacial tension, like the robust pendant drop method, are not suitable for this purpose when the variation of tension lies in the range of accuracy of the method, typically of several percents, mainly depending on the droplet volume [10]. In the same way, the quantification of the settling velocity cannot be used for this because it is sensitive to contamination only in a narrow range of surface concentration [5]. Among the few available techniques, most have been applied to free-surface flows, relying on optical methods such as ellipsometry [11] or reflected second-harmonic generation [12], or based on the attenuation rate of gravity-capillary surface waves [13] or on the fluorescence intensity of a tagged surfactant obtained by stroboscopy [14]. Unfortunately, none of these methods is either easy to carry out with any type of contaminant or to implement within a dispersed two-phase flow. Hosokawa et al. [15] evaluated the surface concentration profile of a rising drop using spatiotemporal filter velocimetry, which gives access to the velocity fields in the vicinity of the interface. This method is however restricted to droplet motion in Stokes regime and is difficult to implement in real flow conditions.

The experimental procedure presented in this Letter only requires a standard high-speed camera able to record sequences of the shape of a moving droplet at a rate of a few thousand Hertz over a second. It provides a simple way to assess the surface concentration of either a droplet or a bubble based on a simple analysis of the shape oscillation dynamics. An initial perturbation of the droplet shape induces oscillations driven by surface tension and 
damped by viscosity. In perfectly clean conditions, wellestablished theoretical works describe the linear shape oscillation dynamics as a series of eigenmodes [16-19] and predict the frequencies and damping rates, provided the effect of gravity can be neglected and the droplet center is immobile. In experiments involving rising droplets in a stagnant liquid, Abi Chebel et al. [20] measured damping rates of oscillation that were significantly higher than theoretical predictions for modes of lower orders but that converged towards predictions for modes of higher orders. The authors suggested that this surprising result could be caused by a subtle coupling between the gradient of interfacial concentration resulting from droplet translation with that generated by shape oscillation. In this Letter, we show that this interpretation is incorrect thanks to comparisons between a direct numerical simulation, which accounts for the effect of surfactants on the rising drop dynamics, and the linear theory, which predicts eigenmodes of shape oscillation by accounting for the Gibbs elasticity of the interface [21] but by neglecting translation. The numerical simulation is first employed to demonstrate that the rising motion does not influence the shape oscillation although it does determine the average profiles of contaminants along the interface. Then, the experimental observations of Abi Chebel et al. [20] are shown to be fully explained by the theory [21], which proves that measuring shape oscillations is a relevant way to assess the surface concentration. This opens the way to reliable in situ determination of the contamination level within experimental laboratory devices or industrial facilities involving dispersed two-phase flows.

Numerical simulation allows to disentangle the effect of gravity from that of interface contamination on the drop dynamics. For a clean droplet, the numerical study of Lalanne et al. [8] revealed that drop translation does not affect the oscillation provided the Weber number We based on the rise velocity remains small. For a contaminated droplet, simulations of Piedfert et al. [22] confirm this conclusion for the oblate-prolate oscillation mode. Here, an oscillating surfactant-ladden droplet of radius $R=$ $0.62 \mathrm{~mm}$ is considered, with an initial shape directly issued from videos of a heptane droplet released in water from a capillary [20], involving several deformation modes at moderate amplitudes (see Fig. 1 and Table II). In the simulation, the drop rise velocity is initialized at zero, with an uniform interface concentration $\Gamma$ of surfactant which is related to the surface tension $\sigma$ by Henry's equation $\sigma=$ $\sigma_{\text {clean }}-\bar{R} T \Gamma$ ( $\sigma_{\text {clean }}$ being the value without surfactants, $T$ the Kelvin temperature and $\bar{R}$ the gas constant). The surfactant is assumed to be insoluble, i.e., the total amount adsorbed at the interface remains constant. The NavierStokes equations are solved on a structured mesh by a finite volume method. The interface is captured by means of the Level-Set method, with a mesh size element of $R / 96$ according to a technique previously validated to simulate

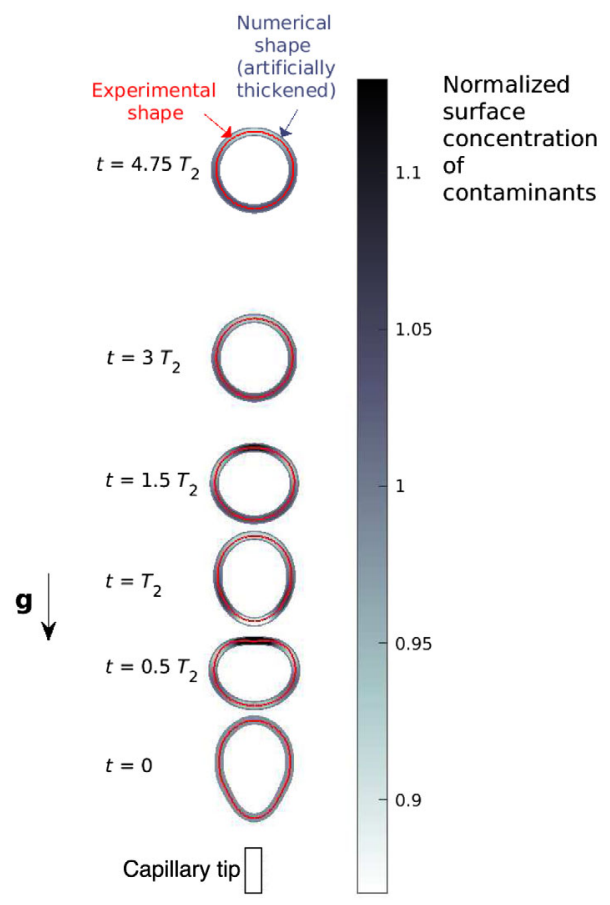

FIG. 1. Computed shapes of an oscillating rising droplet. Time sequence during the transient stage and comparison with the experimental drop shapes (red lines). Color levels correspond to the computed surface concentration of surfactant, normalized by $\Gamma_{\text {eq }}$. Distance between shapes are not to scale. $g$ is the gravity acceleration.

drop dynamics $[22,23]$. The surfactant concentration is computed along the oscillating interface through a convective-diffusion equation [3,24] and the Marangoni stresses rising from gradients of surface concentration are modelled as a discontinuity of the viscous tangential stresses (see Ref. [22] for more details). The drop shape oscillation is simulated during its rise. Both translating and oscillating motions lie in the inertial regime (Reynolds numbers associated to each motion are large, see Table I); the Weber number We is small enough to ensure a quasispherical drop shape at steady state (aspect ratio of 1.01). Figure 1 shows an excellent agreement between experimental and computed shapes during the rise. As the drop accelerates, its shape periodically changes and its rise velocity is shown to oscillate [see Fig. 2(a)] because of periodic variations of the added-mass force acting on the droplet [25]. Surfactant concentration variations at the surface are due to both changes in local interfacial area and advection by the tangential velocity. In particular, the translation strongly influences the interfacial distribution of surfactants by transporting them to the rear of the droplet, within a typical timescale $R / V_{\infty}$ of same order as the oscillation dominant time scale given by the period $T_{2}$ of the $n=2$ oblate-prolate mode: here, $R / V_{\infty} \approx 1.6 T_{2}$. This leads to the development of high surface concentration gradients and Marangoni stresses that slow down the 
TABLE I. Physical parameters for an heptane droplet in water: $\rho_{o}$ and $\rho_{i}$ are the densities of outer and inner phase respectively, $\mu_{o}$ and $\mu_{i}$ their dynamic viscosities, $\sigma_{\mathrm{eq}}$ and $\Gamma_{\mathrm{eq}}$ the interfacial tension and the surface concentration at equilibrium. $\operatorname{Re}_{\mathrm{osc}}=\rho_{o} \sqrt{\left(\sigma_{\mathrm{eq}} / \rho_{o} R^{3}\right)} R^{2} / \mu_{o}$ is the Reynolds number of oscillation, $\operatorname{Re}_{\infty}=2 \rho_{o} V_{\infty} R / \mu_{o}$ the rise Reynolds number at steady state with $V_{\infty}$ the terminal velocity, We $=2 \rho_{o} V_{\infty}^{2} R / \sigma_{\text {eq }}$ the Weber number, $E=\bar{R} T \Gamma_{\text {eq }} / \sigma_{\text {eq }}$ the elasticity number and Ma $=\bar{R} T \Gamma_{\text {eq }} /\left(\mu V_{\infty}\right)$ the Marangoni number.

\begin{tabular}{|c|c|c|c|c|c|c|c|c|c|c|c|c|}
\hline $\begin{array}{l}R \\
(\mathrm{~mm})\end{array}$ & $\begin{array}{c}\rho_{i} \\
\left(\mathrm{~kg} / \mathrm{m}^{3}\right)\end{array}$ & $\begin{array}{c}\rho_{o} \\
\left(\mathrm{~kg} / \mathrm{m}^{3}\right)\end{array}$ & $\begin{array}{c}\mu_{i} \\
\text { (mPa.s) }\end{array}$ & $\begin{array}{c}\mu_{o} \\
\text { (mPa.s) }\end{array}$ & $\begin{array}{c}\sigma_{\text {clean }}, \sigma_{\text {eq }} \\
(\mathrm{mN} / \mathrm{m})\end{array}$ & $\begin{array}{c}\Gamma_{\mathrm{eq}} \\
\left(\mathrm{mol} / \mathrm{m}^{2}\right)\end{array}$ & $\begin{array}{c}g \\
\left(\mathrm{~m} / \mathrm{s}^{2}\right)\end{array}$ & $\operatorname{Re}_{\text {osc }}$ & $\operatorname{Re}_{\infty}$ & $\mathrm{We}_{\infty}$ & $E$ & $\mathrm{Ma}$ \\
\hline 0.62 & 684 & 998.2 & 0.41 & 1.0 & $51.6,47.1$ & $1.86 \times 10^{-6}$ & 9.81 & 171 & 79 & 0.1 & 0.1 & 71 \\
\hline
\end{tabular}

droplet. The drop terminal velocity $V_{\infty}$ is very close to that of a solid particle. As expected from the substantial value of the Marangoni number (around 70), the Marangoni stress is strong enough to eventually cancel the internal drop motion once oscillations are damped. During the transient, the dynamics of the oscillations is analyzed until time $5 T_{2}$ where their amplitudes become negligible and droplet velocity has reached $60 \%$ of $V_{\infty}$. To this purpose, the drop shape is decomposed as a series of eigenmodes, according to Eq. (1):

$$
r(\theta, t)=\sum_{n} a_{n}(t) P_{n}[\cos (\theta)]
$$

where $r$ and $\theta$ are the spherical coordinates of the interface in the frame centered on the droplet center, $a_{n}$ the amplitude of mode $n$ and $P_{n}$ the corresponding Legendre polynomial. Figure 2(a) shows the time evolution of the amplitudes of modes 2 to 5 . Each mode exhibits a clear dominant frequency $\omega_{n}$ and an average damping rate $\beta_{n}$, which are identified through the fit $a_{n}(t)=a_{n}(0) P_{n}[\cos (\theta)] \cos \left(\omega_{n} t\right) \times$ $\exp \left(-\beta_{n} t\right)$. For each mode, $\omega_{n}$ and $\beta_{n}$ are compared to the prediction of the linear theory [21], which neglects gravity and considers small amplitude. Figure 2(b) shows that a remarkable agreement is obtained for both $\omega_{n}$ and $\beta_{n}$ for $n=2-6$, with a discrepancy smaller than $0.5 \%$ for $\omega_{n}$ and $5 \%$ for $\beta_{n}$. Therefore, even though the concentration profile of surfactants at the interface radically differs from that of a nonmoving droplet, the present comparison proves that gravity does not noticeably affect the oscillations. Marangoni stresses associated to translation and oscillation exist simultaneously. However, their roles upon the droplet dynamics are different. The former, dominant in amplitude, is responsible for a decrease of the translation velocity. The latter alone is responsible of a significant increase of the oscillation damping for modes of low order [22]. We can thus conclude that the Lu and Apfel's theory [21] of oscillation, which neglects gravity, can be used to describe the shape oscillation of a contaminated rising droplet, provided oscillation amplitudes are moderate (less than $1 / 5$ of $R$ ) and the average shape is spherical (low We).

Based on the previous conclusions, the experimental results obtained by Abi Chebel et al. [20] are analyzed under the light of Lu and Apfel's theory [21]. In that experiment, heptane droplets of various sizes $(0.3 \leq R \leq 1.8 \mathrm{~mm})$ are formed at a capillary tip and released in water by a mild jolt of the capillary. At the end of the recorded sequenceterminal stage not always reached - the respective ranges of Reynolds and Weber numbers are 20-500 and 0.01-1.4, with an aspect ratio always smaller than 1.12. Since the droplets are initially deformed, damped shape oscillations are observed while the droplets rise. By using a high-speed camera with a magnifying lens, images of $324 \times 864$ pixels of the drop shape evolutions are obtained at a frame rate of order $10^{4} \mathrm{~s}^{-1}$. The fact that the measured drop rise velocities always match that of solid particles supports the assumption of contaminated interfaces. The oscillation time scales are obtained by means of decomposition of drop shapes into spherical harmonics by Eq. (1) (see Ref. [20] for the detailed

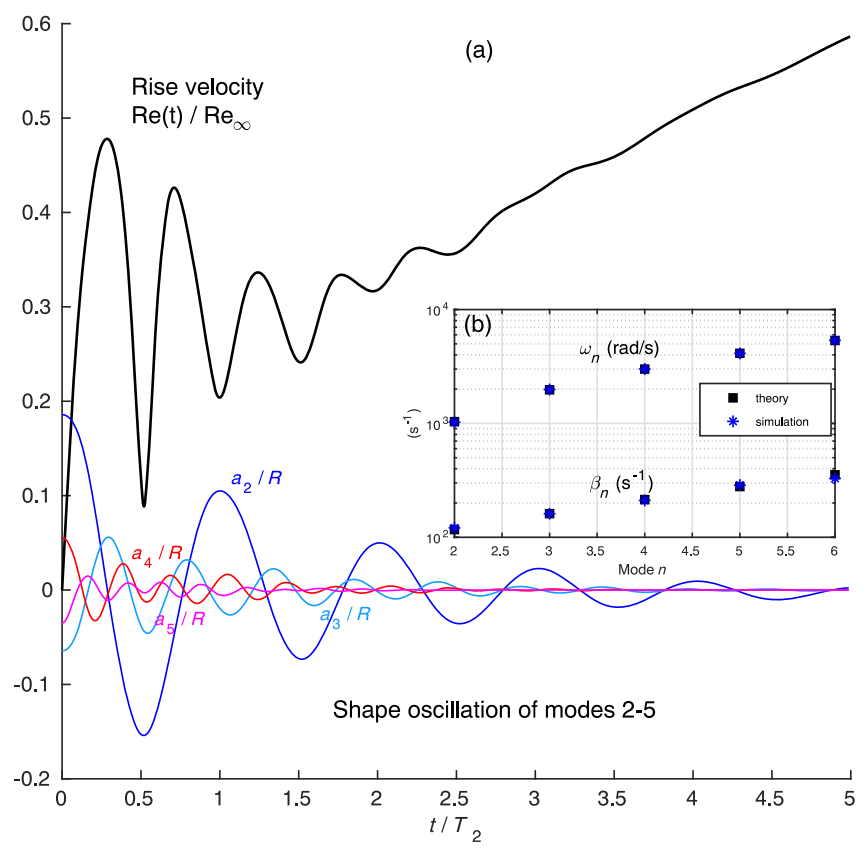

FIG. 2. Simulation results (parameters from Tables I and II). (a) Time evolution of the rise Reynolds number $\operatorname{Re}(t)=$ $2 \rho_{o} V(t) R / \mu_{o}$, with $V(t)$ the instantaneous droplet velocity, normalized by the Reynolds number at terminal stage $\mathrm{Re}_{\infty}$, and of the oscillation amplitudes $a_{n}(t)$ for modes 2-5 normalized by $R$; (b) comparison of angular frequency $\omega_{n}$ and damping rate $\beta_{n}$ between simulation and theory [21]. 
TABLE II. Initial drop shape in the simulation (parameters of Table I), experimentally measured after drop detachment.

\begin{tabular}{lccccccc}
\hline \hline$n$ & 0 & 1 & 2 & 3 & 4 & 5 & 6 \\
\hline$a_{n}(0) / R$ & 0.9912 & 0.0000 & 0.1858 & -0.0644 & 0.0555 & -0.0351 & 0.0273 \\
\hline \hline
\end{tabular}

methodology); frequencies and damping rates of modes 2 to 5 are presented in Fig. 3. Experimental frequencies $\omega_{n \text {,exp }}$ are found in agreement with the theoretical prediction for clean droplets $\omega_{n \text {,clean }}$ [19]. To the contrary, measured damping rates $\beta_{n, \exp }$ are up to 2.5 times larger than the theoretical value for clean droplets $\beta_{n, \text { clean }}$ [19]. In Fig. 3, $\omega_{n, \exp }$ and $\beta_{n \text {,exp }}$ are compared to the theoretical values $\omega_{n, \text { th }}$ and $\beta_{n, \text { th }}$ from the theory [21] that still ignores gravity but accounts
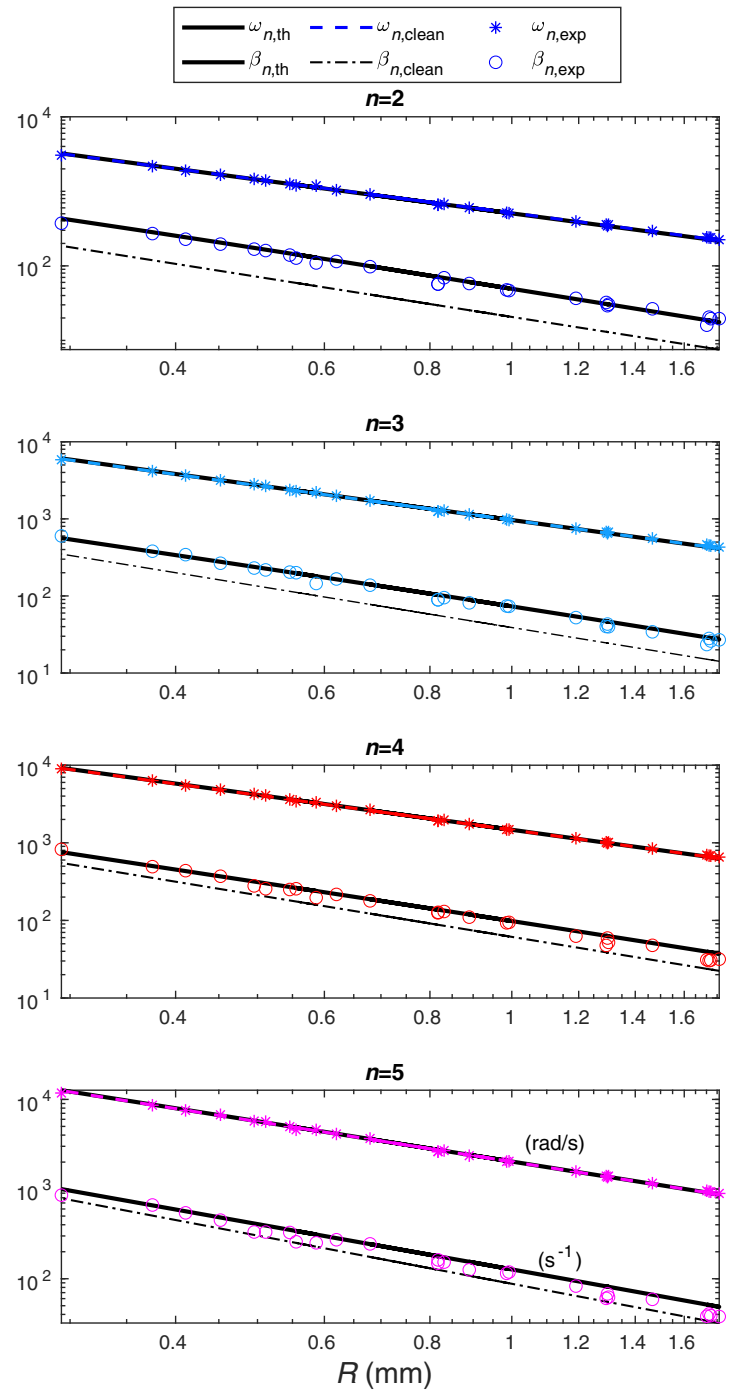

FIG. 3. Experimental values $\omega_{n, \exp }$ and $\beta_{n, \exp }$ for oscillation modes $n=2-5$ from [20], compared to the theoretical predictions $\omega_{n, \text { th }}$ and $\beta_{n, \text { th }}$ from [21] with $\sigma_{\text {eq }}=0.0475 \mathrm{~N} / \mathrm{m}$ and $E=$ 0.1 and to the theoretical values $\omega_{n, \text { clean }}$ and $\beta_{n, \text { clean }}(E=0$, same $\left.\sigma_{\mathrm{eq}}\right)$. for insoluble surfactant effects: $\omega_{n \text {,th }}$ and $\beta_{n \text {,th }}$ are computed by using Eqs. (7)-(9), (17), and (18) from Ref. [21], which are recalled in a Supplementary Material [26]. Note that this theory of shape oscillation, also discussed in $[27,28]$, writes the coupling between linearized NavierStokes and surfactant transport equations. Two unknown parameters are required to compute $\omega_{n, \text { th }}$ and $\beta_{n, \text { th }}$ : the surface tension at equilibrium $\sigma_{\mathrm{eq}}$ and the normalized Gibbs elasticity $E=-\left(\Gamma_{\mathrm{eq}} / \sigma_{\mathrm{eq}}\right)(\partial \sigma / \partial \Gamma)_{\mathrm{eq}}$, where subscript "eq" denotes equilibrium values. In Fig. 3, the values of these parameters have been adjusted to provide the best fit of experimental results: $\sigma_{\mathrm{eq}}=47.5 \pm 1.5 \mathrm{mN} / \mathrm{m}$ and $E=0.1 \pm 0.01$. It leads to predictions of both $\omega_{n \text {,th }}$ and $\beta_{n \text {,th }}$ which are in excellent agreement with all the experimental values from modes 2 to 5 and all droplet sizes. The fact that a unique value of $E$ was identified for all experimental runs suggests that an irreversible adsorption process took place prior to the measurements, making relevant to consider insoluble contaminants. Figure 3 shows that the theory [21] provides the interpretation of the experimental observations of Abi Chebel et al. [20]: the enhancement of the damping rate (relative to $\beta_{n, \text { clean }}$ ) diminishes when the order of the mode increases. Thus, the fact that the model describes all oscillation mode dynamics for all droplets constitutes a strong validation of the overall analysis. Since we consider low surface coverage, it is reasonable to assume a Henry isotherm in order to relate the Gibbs elasticity to the average interface concentration: $E=\bar{R} T \Gamma_{\mathrm{eq}} / \sigma_{\mathrm{eq}}$. Figure 4 shows the evolution of the

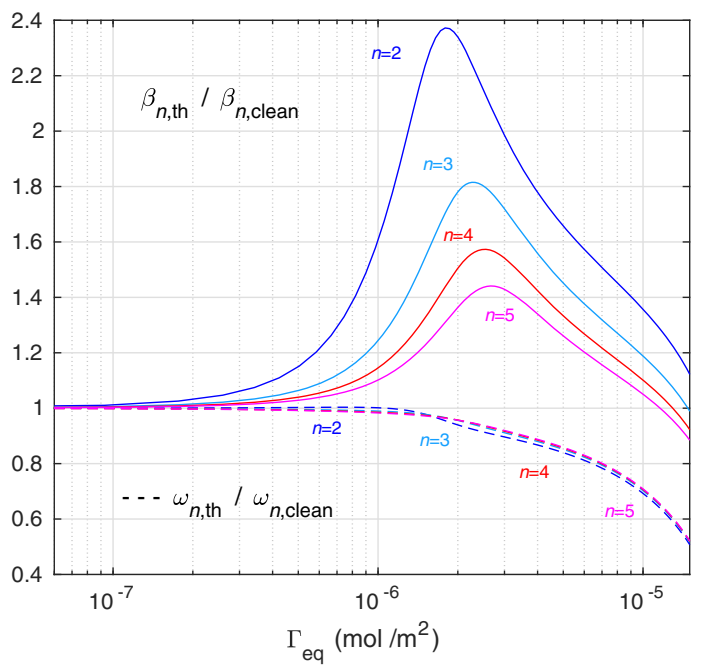

FIG. 4. Evolution of $\omega_{n \text {,th }}$ (dashed lines) and $\beta_{n \text {,th }}$ (continuous lines), normalized by their respective values $\omega_{n \text {,clean }}$ and $\beta_{n \text {,clean }}$, as a function of $\Gamma_{\text {eq }}$. Parameters are in Table I. 
frequency $\omega_{n, \text { th }}$ and the damping rate $\beta_{n, \text { th }}$ when varying $\Gamma_{\text {eq }}$. It turns out that $\omega_{n \text {,th }}$ decreases only slightly when increasing $\Gamma_{\text {eq }}$. Therefore, measuring the frequencies alone cannot lead to an accurate estimate of $\Gamma_{\mathrm{eq}}$ whereas it provides an accurate value of $\sigma_{\text {eq }}$ (like in the study of Ponce et al. [29]). On the other hand, the high sensitivity of $\beta_{n \text {,th }}$ to $\Gamma_{\text {eq }}$ (i.e., to $E$ ), even at low interface coverage, allows an accurate determination of the contamination level from the measurement of the damping rates of first oscillation modes. Applying this procedure to the experimental results of Abi Chebel et al. [20] leads to $\Gamma_{\text {eq }}=1.910^{-6} \pm 0.210^{-6} \mathrm{~mol} / \mathrm{m}^{2}$.

For a contaminated droplet, the present analysis shows that the Marangoni force induced by the gravity-driven motion has a negligible influence on the shape-oscillation dynamics as compared to a nonrising droplet. A strong consequence for experiment design is that microgravity conditions [30] or acoustic trapping [31] are unnecessary to produce shape oscillations that follow the theory [21]: We $\leq 1$ is sufficient for a rising drop or bubble. This allows us to propose a method for measuring the Gibbs elasticity and deducing the surface concentration of surfactants present at low concentration: (1) disturb droplet shape (by release from a capillary tube by a small jet or a mild jolt), (2) record the time evolution of the interface, (3) decompose the shape into spherical harmonics to determine frequencies and damping rates of a few modes, (4) use theory [21] to determine the equilibrium surface tension from frequencies and the Gibbs elasticity from dampings, (5) check that same values are obtained for all modes, and (6) compute surface concentration from Henry's isotherm. The use of such an isotherm is justified for low interface coverage (inducing a surface tension decrease of a few $\mathrm{mN} / \mathrm{m}$ ) and is more suitable for surfactants having short carbon chains [32]. For applicability purpose (low We), we recommend a maximal size of several millimeters for droplets and $d=1 \mathrm{~mm}$ for bubbles. The method works well when $\omega_{2}>\beta_{2}$, allowing for several oscillation periods to be recorded, which is the case with not too highly viscous fluids. This method is simple and can be easily implemented in various experiments. We therefore believe that it can be of great interest for the investigation of many two-phase flows with surface active contaminants at uncontrolled levels, including soft or biological media.

*Benjamin.Lalanne@ensiacet.fr

[1] S. Takagi and Y. Matsumoto, Annu. Rev. Fluid Mech. 43, 615 (2011).

[2] F. H. Garner and A. Hale, Chem. Eng. Sci. 2, 157 (1953).

[3] V. G. Levich, Physicochemical Hydrodynamics (PrenticeHall, Englewood Cliffs, 1962).

[4] R. Clift, J. R. Grace, and M. E. Weber, Bubbles, Drops, and Particles (Academic Press, New York, 1978).
[5] R. Bel Fdhila and P. C. Duineveld, Phys. Fluids 8, 310 (1996).

[6] B. Cuenot, J. Magnaudet, and B. Spennato, J. Fluid Mech. 339, 25 (1997).

[7] K. J. Stebe and C. Maldarelli, J. Colloid Interface Sci. 163, 177 (1994).

[8] B. Lalanne, N. Abi Chebel, J. Vejrazka, S. Tanguy, O. Masbernat, and F. Risso, Phys. Fluids 27, 123305 (2015).

[9] A. Weiner, J. Timmermann, C. Pesci, J. Grewe, M. Hoffmann, M. Schlüter, and D. Bothe, Chem. Eng. Sci. 1, 100007 (2019).

[10] J. D. Berry, M. J. Neeson, R. R. Dagastine, D. Y. Chan, and R. F. Tabor, J. Colloid Interface Sci. 454, 226 (2015).

[11] J. Hutchison, D. Klenerman, S. Manning-Benson, and C. Bain, Langmuir 15, 7530 (1999).

[12] M. J. Vogel, A. H. Hisa, J. S. Kelley, and G. M. Korenowski, Rev. Sci. Instrum. 72, 1502 (2001).

[13] J. R. Saylor, A. J. Szeri, and G. P. Foulks, Exp. Fluids 29, 509 (2000).

[14] S. L. Strickland, M. Shearer, and K. E. Daniels, J. Fluid Mech. 777, 523 (2015).

[15] S. Hosokawa, Y. Masukura, K. Hayashi, and A. Tomiyama, Int. J. Multiphase Flow 97, 157 (2017).

[16] J. W. S. Rayleigh, Proc. R. Soc. Lond. 29, 71 (1879).

[17] H. Lamb, Hydrodynamics (Cambridge University Press, Cambridge, 1932).

[18] C. A. Miller and L. E. Scriven, J. Fluid Mech. 32, 417 (1968).

[19] A. Prosperetti, J. Méc. 19, 149 (1980).

[20] N. Abi Chebel, J. Vejrazka, O. Masbernat, and F. Risso, J. Fluid Mech. 702, 533 (2012).

[21] H. L. Lu and R. Apfel, J. Fluid Mech. 222, 351 (1991).

[22] A. Piedfert, B. Lalanne, O. Masbernat, and F. Risso, Phys. Rev. Fluids 3, 103605 (2018).

[23] B. Lalanne, L. R. Villegas, S. Tanguy, and F. Risso, J. Comput. Phys. 301, 289 (2015).

[24] H. Stone, Phys. Fluids A 2, 111 (1990).

[25] B. Lalanne, S. Tanguy, and F. Risso, Phys. Fluids 25, 112107 (2013).

[26] See the Supplemental Material at http://link.aps.org/ supplemental/10.1103/PhysRevLett.124.194501 for a practical form of the procedure to compute $\omega_{n, \text { th }}$ and $\beta_{n, \text { th }}$, summarized from Ref. [21].

[27] Y. Tian, R. G. Holt, and R. E. Apfel, J. Colloid Interface Sci. 187, 1 (1997).

[28] N. Abi Chebel, A. Piedfert, B. Lalanne, C. Dalmazzone, C. Noik, O. Masbernat, and F. Risso, Langmuir 35, 9441 (2019).

[29] A. Ponce-Torres, J. M. Montanero, M. A. Herrada, E. J. Vega, and J. M. Vega, Phys. Rev. Lett. 118, 024501 (2017).

[30] R. E. Apfel et al., Phys. Rev. Lett. 78, 1912 (1997).

[31] T. J. Asaki, D. B. Thiessen, and P. L. Marston, Phys. Rev. Lett. 75, 2686 (1995).

[32] D. F. Evans and H. Wenneström, The Colloidal Domain: Where Physics, Chemistry, Biology, and Technology Meet (Wiley-VCH, New York, 1999). 